Draft VERSION JunE 1, 2021

Preprint typeset using $\mathrm{LAT}_{\mathrm{E}} \mathrm{X}$ style emulateapj v. 5/2/11

\title{
AGE AND MASS SEGREGATION OF MULTIPLE STELLAR POPULATIONS IN GALACTIC NUCLEI AND THEIR OBSERVATIONAL SIGNATURES
}

\author{
Hagai B. Perets and Alessandra Mastrobuono-Battisti \\ Draft version June 1, 2021
}

\begin{abstract}
Nuclear stellar cluster (NSCs) are known to exist around massive black holes (MBHs) in galactic nuclei. They are thought to have formed through in-situ star formation following gas inflow to the nucleus of the galaxy and/or through the infall of multiple stellar clusters. Here we study the latter, and explore the composite structure of the NSC, and its relation to the various stellar populations originating from its progenitor infalling clusters. We use N-body simulations of clusters infall, and show that this scenario may produce observational signatures in the form of age segregation: the distribution of the stellar properties (e.g. stellar age and/or metallicity) in the NSCs reflect the infall history of the different clusters. The stellar populations of clusters infalling at different times (dynamical ages), are differentially segregated in the NSC, and are not fully mixed even after few Gyrs of evolution. Moreover, the radial properties of stellar populations in the progenitor cluster are mapped to their radial distribution in the final NSC, potentially leading to efficient mass segregation in NSCs, even those where relaxation times are longer than a Hubble time. Finally, the overall structures of the stellar populations present non-spherical configurations and show significant cluster to cluster population differences.
\end{abstract}

\section{INTRODUCTION}

Nuclear stellar clusters (NSCs) hosting massive black holes (MBHs) are thought to exist in a significant fraction of all galactic nuclei. The build-up of such dense clusters is likely linked to the growth of the $\mathrm{MBH}$, and the evolution of the galaxy and its nucleus, as suggested by statistical correlations between their properties (e.g. Ferrarese \& Merritt 2000; Tremaine et al. 2002). Two scenarios were suggested for the origin of nuclear clusters: (1) The cluster infall scenario, in which stellar clusters inspiral to the galactic nucleus, disrupted, and thereby build up the nuclear cluster (bek + Tremaine et al. 1975; Capuzzo-Dolcetta 1993; Bekki et al. 2004; Agarwal \& Milosavlievió 2011; Antonini et al. 2012; Antonini 2013; Gnedin et al. 2013 and references therein) the inspiraling clusters may also be the NSC of two galaxies in a merger scenario). (2) The nuclear star formation scenario, in which gas infalls into the nucleus and then transforms into stars through star formation processes (Loose et al. 1982; possibly in a disk like configuration, e.g. observations of the Milky Way NSC; Levin \& Beloborodov 2003; Lu et al. 2009; Bartko et al. 2009). Over time the in-situ star formation builds up the NSC. Naturally, both processes can work in concert, and both could be important for the formation and evolution of NSCs.

In this paper we explore the cluster infall scenario by means of N-body simulations, and neglect star formation processesand their effects, which are beyond the scope of this paper. Previous studies have dealt with the global structure and build-up of NSCs from inspiral of globular clusters (GCs). Here we focus on the multiple stellar populations in NSC and their mapping and relations to their original host GCs. We study the evolution of the multiple GC populations both during the evolution of the NSC and in its final form. In particular, we show that the cluster infall scenario introduces population segre- gation in NSCs, and provides signatures of the cluster infall history in the radial distribution of the stars in the NSC. In addition we suggest that the formation of NSCs from cluster infall can produce mass segregated NSCs, even in cases where two-body relaxation processes are too slow. We suggest that such variations in the kinematic properties of different stellar populations might be observable and serve as fossilized evidence for the evolution and build-up of galactic nuclei.

In the following we begin by a brief description of our cluster infall scenario (described in more details in Paper I). We then present the distribution of the stellar populations of the different infalling cluster in the fully formed NSC, and show the existence of the age-segregation and mass segregation phenomena. Finally, we discuss our results, discuss their implications and summarize.

\section{SIMULATION OF THE CLUSTER INFALL FORMATION OF A NUCLEAR CLUSTER}

Our modeling of the cluster infall formation of an NSC follows the same methods, and make use of the same code as used in paper I (Antonini et al. 2012), where detailed description of the initial conditions of GCs and the galaxy model of the background stellar population can be found. In brief, we used direct N-body simulations (using the $\phi$ GRAPE code Harfst et al. 2007) of the consecutive infall and merging of a set of 12 single-mass globular clusters each starting from a galactocentric distance of $20 \mathrm{pc}$. One simplification that we make is taking a constant time interval between cluster infalls, as done in previous works (e.g. Paper I). The total mass of these clusters sums to $\sim 1.5 \times 10^{7} M_{\odot}$, which is roughly the observed mass of the Milky Way nuclear star cluster (Genzel et al. 2010). The mass of the GCs is comparable to currently observed Milky Way GCs (see Gnedin \& Ostriker 1997, for MilkyWay GC paramters), though a better comparison would be to young superclusters (or their leftover nuclei) that would have inspiarlled to the nucleus (e.g. Kroupa 1998), 
and are currently observed only in other galaxies (e.g. young super star-clusters such as R136 observed in the LMC). with a $\mathrm{MBH}$ of $4 \times 10^{6} \mathrm{M}_{\odot}$. After the first cluster had spiraled in to the center, we let the system reach a nearly steady state (as evaluated via Lagrange radii), and then added a second cluster. We iterated this procedure until all clusters accumulated and merged to form an NSC around the central MBH. We note a few differences from the original simulations discussed in paper I: (1) The scaling of the relaxation time used in paper I assumed a fully collisional simulation, where as in effect a softening radius of $0.01 \mathrm{pc}$ is used; we now scale the relaxation times correctly, accounting for the lower cutoff of the Coloumb logarithm due to the softening radius used. The times used in paper I were therefore 2.9 times shorter than the now corrected estimates. (2) We now assume a random distribution of the initial inclinations and phases of the inspiraling clusters, rather than a the contrived model in paper I; the initial conditions are shown in Table 1. (3) The total scaled time of the simulation is 12 Gyrs, comparable to the age of the Galaxy; the time passed since the infall of the last cluster until the end of the simulation is shorter than the relaxation time of the NSC, but we have also followed the simulation up to the relaxation time of the NSC. We emphasize that the times used here are only an approximation, based on scaling arguments of the relaxation time, and should not be treated as an accurate time representation.

We have made two realizations of the infall scenario for the formation of the NSC. Both realizations show generally similar results, and we present results only from one of them (see Table 1 for the relevant initial conditions). We briefly remark on some differences observed between the two realizations.

\section{RESULTS}

In this study we explore the mapping between the properties of stellar populations in NSC, and their relation to the initial characteristics of the stellar populations in the progenitor clusters. We focus on two points in time during the NSC evolution; the first after the infall and initial relaxation of the last cluster, and the second, after more relaxation has occurred at an age comparable to a Hubble time. Our findings show that the dynamical history of the cluster infall is still reflected in the radial distribution of the stellar populations in the NSC, even

\begin{tabular}{cccc}
\hline $\mathrm{n}$ & $\Omega(\mathrm{deg})$ & $i(\mathrm{deg})$ & $R_{t}(\mathrm{pc})$ \\
\hline 1 & 82.4 & 60.7 & 1.29 \\
2 & 327.7 & 178.7 & 1.29 \\
3 & 76.2 & 139.5 & 1.29 \\
4 & 290.6 & 171.3 & 1.34 \\
5 & 335.4 & 24.6 & 1.48 \\
6 & 300.6 & 18.2 & 1.54 \\
7 & 343.9 & 173.9 & 1.55 \\
8 & 47.9 & 128.9 & 1.6 \\
9 & 272.0 & 2.3 & 1.78 \\
10 & 41.3 & 139.0 & 1.80 \\
11 & 300.9 & 153.5 & 1.85 \\
12 & 318.2 & 120.2 & 1.86 \\
\hline
\end{tabular}

TABLE 1

INITIAL ORBITAL PARAMETERS OF THE INFALLING CLUSTERS (INCLINATION I AND LONGITUDE OF ASCENDING NODE $\Omega$ ), AND THEIR TIDAL DISRUPTION RADIUS AS FOUND FROM THE SIMULATIONS.
Gyrs after the last infall.

We find that stars in the NSC originating from earlyinfall clusters are more segregated to the center of the NSC than their stellar counterparts from late-infall clusters. This is true both after the last infall and even later after a few Gyrs of evolution (See Fig. 1). In particular the cluster inner population (central hundred particles), show a clear segregation when comparing early and late infalls, with relatively little additional mixing over the last two Gyrs of evolution (See the evolution of the Lagrangian radii of these populations in the NSC in Fig. 2). Note that the age segregation is reversed between the inner regions of the NSC and the outer regions, due to the later (earlier) stripping of the early (late)-infalling clusters.

As can be seen in Fig. 2, the infall of each new cluster affects the evolution of the stellar population of the previous infalling cluster, effectively "compressing" it into a more compact configuration around the $\mathrm{MBH}$. The last infalling cluster did not experience such a later infall, and the distribution of its stars is significantly less segregated. Observing such a distinct population in a galactic nucleus could therefore provide an interesting clue on a relatively recent infall. The more robust results apply for the earlier 11 clusters, in which the age segregation signatures can be observed even long after their infall.

We also find that the 3D structure of the stellar populations of each of the GCs could significantly differ. In Fig. 3 we show the triaxiality parameter (see Paper I) for each of the GC populations as a function of the distance from the $\mathrm{MBH}$. As can be seen, such structure could vary significantly even between consecutive infalling clusters.

We note that some bunching of the population of several consecutive clusters can be observed (e.g. GCs 1-4, then GCs 5-8, and then 9-11). This bunching relates to the specific initial conditions for each cluster (i.e. its inclination and orbital phase; $\mathrm{i}$ and $\Omega$ ), and different bunching is observed when different random initial conditions are used (not shown). However, this interesting phenomena do not affect the overall age and mass segregation processes discussed here, and are beyond the scope of this paper.

\section{DISCUSSION}

\subsection{Age segregation}

An additional aspect of NSC build-up from cluster infall is the mapping of the infalling clusters structure to the final configuration of the NSC. Typically stars at a distance $R_{c}$ (which contains $M\left(<R_{c}\right.$ ) of the cluster mass) from the center of their infalling host cluster are stripped by the tidal forces due to the combined mass of the MBH and the stellar mass of the NSC, $M_{N S C}$ at the stripping radius, $R_{s}$, defined as the tidal radius for stars at that position:

$$
R_{s}=\left(\frac{M_{B H}+M_{N S C}\left(<R_{s}\right)}{M_{c}\left(<R_{c}\right)}\right)^{1 / 3} R_{c} .
$$

At early stages, the NSC mass is dominated by the $\mathrm{MBH}\left(M_{B H} \gg M(<R)\right)$ and the early falling clusters should have very similar tidal radii. At later stages the 

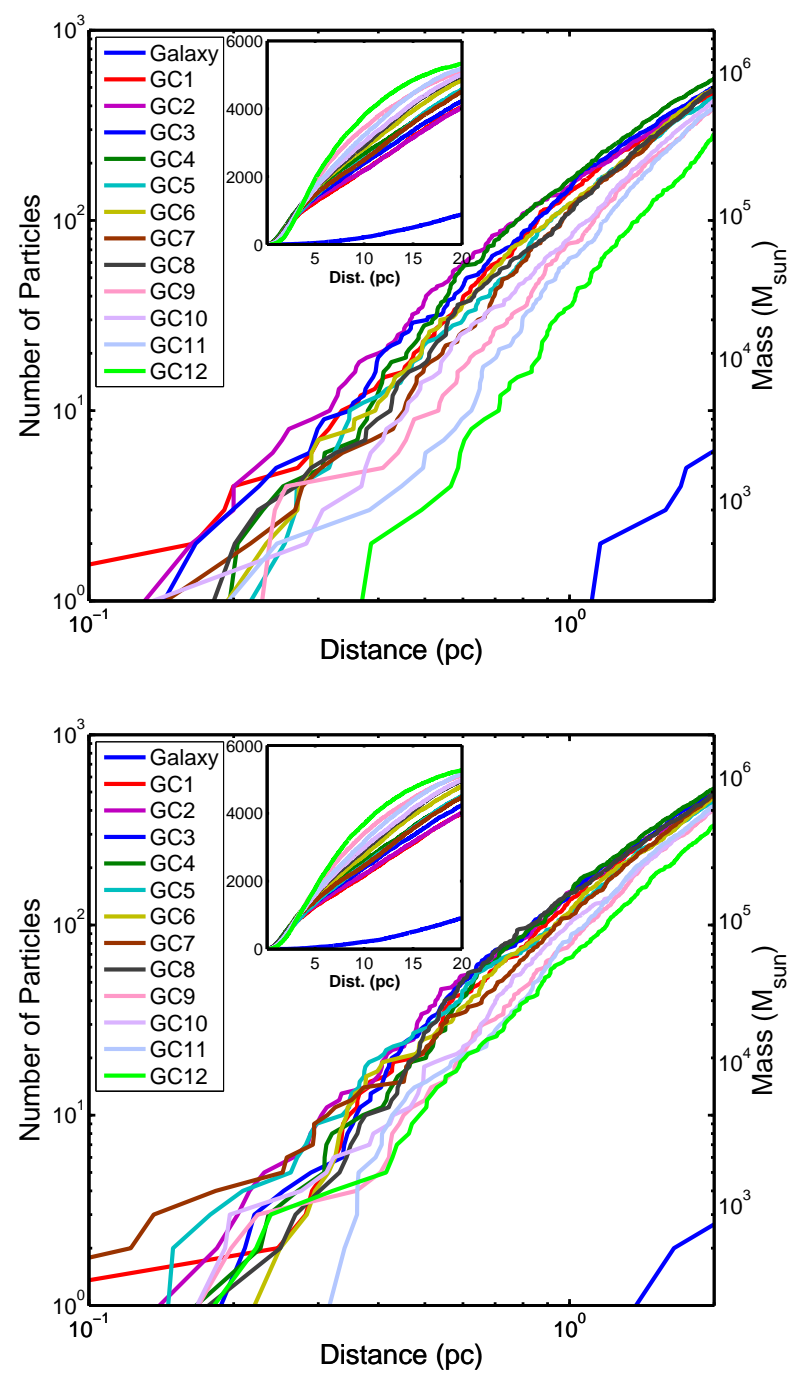

FIG. 1.- Radial distribution of the infalling clusters stellar populations. Top: the radial distribution following the last cluster infall (after the initial relaxation). Bottom: The same at 12 Gyrs. As can be seen in both cases the clusters populations show clear differences, with the earlier infalling clusters showing systematically more compact configuration in the central region $(<2 \mathrm{pc})$, and then an opposite behavior outside. Insets show the large scale (20 pc) distribution; note linear scales in insets.

NSC is slowly built up from the infalling clusters, until it reaches a mass comparable and even larger than the $\mathrm{MBH}\left(M_{N S C} \sim 2-3 \times M_{B H}\right)$. We therefore expect stars from later clusters to be stripped earlier, at a stripping radius which is up to $2^{1 / 3}-3^{1 / 3}(1.26-1.44)$ larger than the corresponding stripping radius for stars from earlier GCs (i.e. initially at comparable distances from the GC center in both clusters). This expectation is consistent with our results in table 1 and Fig. 2. indeed the first infalling clusters show the same tidal radii, and the ratio between the tidal radii of the last and the first infalling clusters is $1.86 / 1.29 \simeq 3^{1 / 3}$. This phenomenon produces a dynamical age gradient, where stars from earlier infalling clusters are stripped at later stages, and dominate inner regions of the NSC. If earlier falling clusters are older (e.g. they formed earlier and therefore spiraled earlier to the nucleus) then such gradient would be translated into a stel-

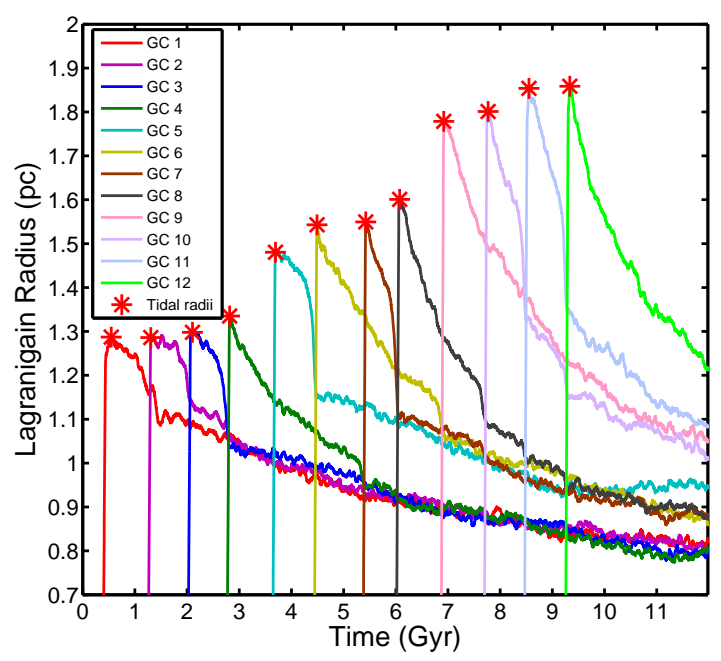

FIG. 2.- Evolution of the Lagrangian radii for the central hundred stars of each of the clusters. The initial Lagrange radius is 0.2 pc for each cluster. Once the cluster is disrupted (see star symbols), the Lagrange radius transitions from being relative to the location of the central density of the cluster to effectively become the distance from the $\mathrm{MBH}$, hence the rise from $0.2 \mathrm{pc}$ (no seen) to the tidal radius central density of each cluster. As can be clearly seen, the stellar population of the earlier falling clusters is progressively more centrally concentrated than the populations of later falling clusters.

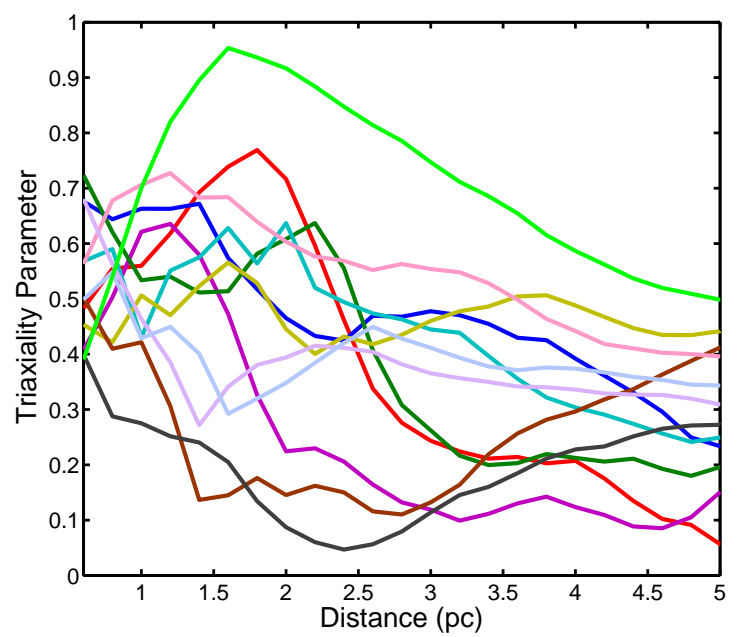

FIG. 3.- Triaxiality of the NSC stellar populations. The triaxiality parameter of the different cluster populations (smoothed for clarity with a $1 \mathrm{pc}$ window) is shown as a function of distance from the MBH. As can be seen the different populations show distinctly different behavior, and significant triaxiality. In particular, some of the cluster show highly anisotropic properties; somewhat resembling thick-disk-like structures in some cases even Gyrs after their infall.

lar age gradient. Alternatively, if earlier falling clusters differ in metallicity/color than their later counterparts (e.g. later falling cluster arrive from larger distances where colors differ; e.g. Balcells \& Peletier 1994 ), such gradient would be translated into a color/metallicity gradient in the NSC. The dynamical age segregation process may therefore give rise to potentially observable stellar age/metallicity gradients. 
We do note an important complication with this simplified picture. In particular, more compact and massive GCs will be stripped later (see Eq. 1), and may therefore dispose their stars closer to the $\mathrm{MBH}$, compared with the stars of the earlier, more dispersed clusters. Note, however, that less compact clusters are less likely to inspiral to the nucleus from large distances during a Hubble time (see Antonini et al. 2012), and therefore we expect only the most dense massive clusters to contribute to the stellar population in the NSC. Moreover, given the shorter inspiral time of more massive clusters, these are likely to arrive earlier, on average, than their lighter counterparts, which would also contribute to their later stripping.

\subsection{Mass segregation}

Irrespective of the age segregation process discussed above, the mapping between the structure of the infalling GC and the structure of the stripped population of stars in the NSC suggested by Eq. 1 could have an important role in building mass segregated NSCs. It is thought that massive clusters are likely to be mass segregated either primordially or from an early stage in their evolution e.g. Baumgardt et al. 2008; Allison et al. 2009; Portegies Zwart et al. 2010, and references therein; and in even in the absence of such early mass segregation, GCs may have sufficient time to segregate during their infall. Such mass segregation could potentially be mapped into a mass segregation of galactic nuclei stellar population. Indeed, the locations of stars initially outside the GC cores are strongly correlated with their positions in the galaxy nucleus at the end of the simulation (linear correlation coefficients, $R$, almost monotonically rise between the first infalling clusters, $R=0.48$ to the last infalling cluster, $R=0.18$ ). Note, however, that we find such direct mapping of closer in stars do not hold for stars initially inside the GC cores. These stars would still be more centrally concentrated around the $\mathrm{MBH}$ at the end of the simulation than stars initially residing in the outer parts of the GCs (by a factor of 2-3), but among these core stars any direct correlation between their initial position in the GC and their final position in the galaxy nucleus is lost.

The infall scenario would therefore suggest that NSCs are likely to be mass segregated even when the relaxation times in such NSCs are longer than a Hubble time (true for most NSCs hosting MBHs with $M_{B H}>$ few $\left.\times 10^{7}\right)$. More generally, NSCs could be more mass segregated than expected from typical relaxation processes (e.g. Bahcall \& Wolf 1977; though strong mass segregation process may also contribute; Alexander \& Hopman 2009). As a side note, it is interesting to point out that clues for an extreme mass segregation are apparent in the stellar population of the Galactic NSC (see Alexander 2007 for a discussion).

Such dynamical-mapping age segregation could have an important role in leading to the concentration of massive stars, and in particular stellar black holes in the central region of nuclear cusps around MBHs. The built-up of a centrally concentrated dark cusp made of black holes is therefore an interesting potential outcome of the cluster infall scenario for NSC formation (see Antonini 2014, in prep. for a detailed discussion of these issues). We also note that in cases an infalling GC harbors an intermediate mass black hole $\left(10^{3}-10^{4} \mathrm{M}_{\odot}\right)$, it could bring stars much closer in to the NSC MBH, thereby producing an even more compact configuration (see Mastrobuono and Perets, in prep. for such a scenario).

\subsection{Relaxation time vs. mixing time}

Following the cluster infall formation of NSC, they may continue to evolve through two-body relaxation processes. In theory, such later evolution may progressively erase some or all of the signatures of the cluster infall scenario discussed above given sufficient time for evolution. However, as shown above, we find that signatures of the infall scenario are observed even at the age of the universe. Moreover, even at later times, comparable to the relaxation time of the NSC after the last infall, the NSC still shows clear signatures of the dynamicalmapping age and mass segregation. This may appear counter intuitive, as one might expect any initial conditions in the cluster to be erased after a relaxation time. Relaxation time is defined as the time it takes a star to change its kinetic energy by the order of itself (e.g. Binney \& Tremaine 1987). However, in order for a star in the outer region of an NSC to be transported to the inner region of the NSC, it needs a much more significant change in energy, i.e. of the order of the energy of the star in the inner region, were the gravitational potential and velocities are much higher. As a first simplified approximation for the time it takes a stellar population at some distance $r_{\text {out }}$ from MBH to mix with another stellar population at an inner region, $r_{i n}$, one can replace the velocity dispersion in relaxation time formula at the position $r_{\text {out }}$ with that in the position $r_{i n}$ while keeping the number densities and the relative velocities between stars, the same i.e. the "mixing time" for the two populations would be defined by

$$
t_{\text {mix }\left(r_{\text {out }}, r_{\text {in }}\right)}=\left(\frac{\sigma\left(r_{\text {in }}\right)^{2}}{\sigma\left(r_{\text {out }}\right)^{2}}\right) t_{r_{\text {out }}} \approx\left(\frac{r_{\text {out }}}{r_{\text {in }}}\right) t_{r_{\text {out }}},
$$

where $t_{r_{\text {out }}}$ is the relaxation time at $r_{\text {out }}$ and the last equality is obtained for regions where the $\mathrm{MBH}$ dominates the gravitational potential of the NSC (i.e. up to the $\mathrm{MBH}$ influence radius). A more accurate definition would account for the changing diffusion time as the stellar environment changes during the diffusive transport of a star from one environment to another; a full discussion of the mixing time is beyond this scope and will be discussed in details elsewhere. Irrespective of the accurate definition, it is clear that $t_{m i x}>t_{r}$; stellar population segregation could therefore survive much longer than a relaxation time. One should note, however, that every infall of an additional cluster does not only bring new stars to the NSC, that would slowly change the two-body relaxation time, but can produce significant changes in the gravitational potential on dynamical timescales. Though this may not significantly affect the inner regions deep in the potential of the $\mathrm{MBH}+\mathrm{NSC}$, such changes may give rise to a more violent relaxation in the outer regions that can mix the stellar populations much more efficiently than two-body relaxation processes. One would therefore expect segregated populations to be more pronounced in the inner regions of NSCs, and around more massive MBHs.

\subsection{Structure of the multiple stellar populations}


The particular final structure of the stellar population of each GC is complex, as can be seen in Fig. 3. and its detailed exploration is beyond the scope of this letter. Here we only note that the significant differences between these structures could provide an additional signature for the multi-cluster infall scenario, similar to the radial segregation discussed above. In particular infalling clusters can produce thick flattened structures with varied orientations, possibly related to "disky" like structures are observed in galactic nuclei and clusters (see Mastrobuono-Battisti \& Perets 2013 for discussion of the evolution of such disks).

\section{SUMMARY}

In this letter we explore the signatures and the implications of the cluster infall scenario on the structure of nuclear stellar clusters and their multiple stellar populations. We use N-body simulations to study the infall of 12 globular clusters into a galactic nucleus hosting a $\mathrm{MBH}$ of $4 \times 10^{6} \mathrm{M}_{\odot}$, and we follow the evolution of the stellar populations from each cluster and their final distribution in the NSC. We find that the infall history is reflected in the final structure of the NSC, where stellar populations from earlier falling clusters are more concentrated in the central parts of the NSC compared to late ones. This dynamical age segregation process can potentially leave behind a signature in the form of an age and/or metallicity radial gradient in the NSC stellar population. The stellar population of each cluster forms a non-spherical complex structure, which behavior significantly differs from one cluster population to another. In addition, any primordial/early mass segregation in the infalling GCs is mapped into a mass segregated populations in the galactic nucleus; in particular even NSCs where relaxation time is longer than a Hubble time could show a mass segregated stellar population, which could not arise from two-body relaxation processes.

We would like to thank Fabio Antonini for for helpful discussions and comments. We would also like to thank the referee for helpful comments and suggestions that improved this manuscript. We acknowledge support from the I-CORE Program of the Planning and Budgeting Committee and The Israel Science Foundation grant $1829 / 12$. HBP is a Deloro and BIKURA fellow. AMB is partly supported by the Lady Davies Foundation.

\section{REFERENCES}

Agarwal, M. \& Milosavljević, M. 2011, ApJ, 729, 35

Alexander, T. 2007, arXiv:0708.0688

Alexander, T. \& Hopman, C. 2009, ApJ, 697, 1861

Allison, R. J., Goodwin, S. P., Parker, R. J., de Grijs, R. Portegies Zwart, S. F., \& Kouwenhoven, M. B. N. 2009, ApJ, 700, L99

Antonini, F. 2013, ApJ, 763, 62

Antonini, F., Capuzzo-Dolcetta, R., Mastrobuono-Battisti, A., \& Merritt, D. 2012, ApJ, 750, 111

Bahcall, J. N. \& Wolf, R. A. 1977, ApJ, 216, 883

Balcells, M. \& Peletier, R. F. 1994, AJ, 107, 135

Bartko, H., Martins, F., Fritz, T. K., Genzel, R., Levin, Y. Perets, H. B., Paumard, T., Nayakshin, S., Gerhard, O., Alexander, T., Dodds-Eden, K., Eisenhauer, F., Gillessen, S., Mascetti, L., Ott, T., Perrin, G., Pfuhl, O., Reid, M. J., Rouan, D., Sternberg, A., \& Trippe, S. 2009, ApJ, 697, 1741

Baumgardt, H., De Marchi, G., \& Kroupa, P. 2008, ApJ, 685, 247

Bekki, K., Couch, W. J., Drinkwater, M. J., \& Shioya, Y. 2004, ApJ, 610, L13

Binney, J. \& Tremaine, S. 1987, Galactic Dynamics (Princeton, NJ: Princeton University Press)

Capuzzo-Dolcetta, R. 1993, ApJ, 415, 616
Ferrarese, L. \& Merritt, D. 2000, ApJ, 539, L9

Genzel, R., Eisenhauer, F., \& Gillessen, S. 2010, Reviews of Modern Physics, 82, 3121

Gnedin, N. Y. \& Ostriker, J. P. 1997, ApJ, 486, 581

Gnedin, O. Y., Ostriker, J. P., \& Tremaine, S. 2013, arXiv: 1308.0021

Harfst, S., Gualandris, A., Merritt, D., Spurzem, R., Zwart, S. P., \& Berczik, P. 2007, New Astronomy, 12, 357

Kroupa, P. 1998, MNRAS, 300, 200

Levin, Y. \& Beloborodov, A. M. 2003, ApJ, 590, L33

Loose, H. H., Kruegel, E., \& Tutukov, A. 1982, A\&A, 105, 342

Lu, J. R., Ghez, A. M., Hornstein, S. D., Morris, M. R., Becklin, E. E., \& Matthews, K. 2009, ApJ, 690, 1463

Mastrobuono-Battisti, A. \& Perets, H. B. 2013, ApJ, 779, 85

Portegies Zwart, S. F., McMillan, S. L. W., \& Gieles, M. 2010 , ARA\&A, 48, 431

Tremaine, S. et al. 2002, ApJ, 574, 740

Tremaine, S. D., Ostriker, J. P., \& Spitzer, Jr., L. 1975, ApJ, 196, 407 